\title{
Flotación por aire disuelto con coagulante policloruro de aluminio para aguas residuales de industrias avícolas
}

\section{Dissolved air flotation with polyaluminum chloride coagulant for wastewater from poultry industries}

\section{Yaxcelys Caldera}

yaxcelysc@gmail.com

Código ORCID: 0000-0002-4862-9313

Universidad del Zulia.Maracaibo, Venezuela

\author{
Mayra Sánchez \\ mayravsanchezc@gmail.com \\ Código ORCID: 0000-0002-6800-724X \\ Universidad del Zulia. Cabimas, Venezuela
}

\author{
Edixon Gutiérrez \\ egutierr12@gmail.com \\ Código ORCID: 0000-0002-9468-3644 \\ Universidad del Zulia. Cabimas, Venezuela
}

\section{Resumen}

Se evalua la eficiencia del coagulante policloruro de aluminio (PAC) en un sistema de flotación por aire disuelto (DAF) durante el tratamiento de aguas residuales de una industria avícola (ARIA). Se trabaja con un equipo de DAF de 4 I en la cámara de flotación. Se determinaron los parámetros $A$ y G, DQO y SST, antes y después del tratamiento, a presiones de 30, 40 y 50 psi, porcentajes de recirculación del efluente de 20, 30 y $40 \%$, sin coagulante y con la adición de PAC. El sistema de DAF fue eficiente para remover entre 87 y $97 \%$ de $A$ y $G$ de las ARIA, con y sin la adición de PAC. El coagulante mejora la eficiencia del sistema de DAF para la remoción de DQO y SST, obteniendo máximos de 90 y $79 \%$, respectivamente.

Palabras clave: Aguas residuales, coagulante, DAF, flotación, industria avícola, policloruro de aluminio

\begin{abstract}
The efficiency of the coagulant polyaluminum chloride (PAC) in a system of dissolved air flotation (DAF) was evaluated during the treatment of poultry industry wastewater (WPI). We worked with a DAF 4 I in the float chamber. Parameters O \& G, COD and TSS, before and after treatment were determined, at pressures of 30, 40 and 50 psi, effluent recirculation percentages 20,30 and $40 \%$, without coagulating and with the addition of PAC. DAF system was efficient to remove $O \& G$ between 87 and $97 \%$ of the WPI, with and without the addition of PAC. The coagulant improved the efficiency of the DAF system for the removal of COD and TSS, obtaining maxim of 90 and $79 \%$, respectively.
\end{abstract}

Keywords: Wastewater, coagulant, DAF, flotation, poultry industry, polyaluminum chloride 


\section{LISTA DE ABREVIATURAS}

\begin{tabular}{ll}
\hline A y $G$ & Fósforo, nitrógeno, aceites y grasas \\
ARIA & Residuales de una industria avícola \\
DAF & Flotación por aire disuelto \\
DQO & Demanda química de oxígeno \\
PCA & Policloruro de aluminio \\
SS & Sólidos suspendidos \\
\hline
\end{tabular}

\section{INTRODUCCIÓN}

En la agroindustria se incluyen las plantas de beneficio animal, cuya operatividad requiere inocuidad en el manejo del producto para abastecimiento de la población, así como la garantía del cuidado del medio ambiente. Dentro de las plantas de beneficio animal, están contenidas las del sector avícola, cuyas áreas son: la recepción del animal, sacrificio y desplume, evisceración, escurrido y empaque, preparación para la comercialización y otras áreas del proceso, donde se debe cumplir con los estándares sanitarios y ambientales, en la búsqueda de prevenir o minimizar los efectos negativos sobre el entorno y los operarios, mantener la limpieza y ofrecer productos finales con la frescura y calidad exigida por los consumidores (Fernández y Betancourt, 2018, Meleán y col., 2008).

De esta manera, en las distintas etapas del proceso productivo de las industrias avícolas se requiere un gran volumen de agua, principalmente en el lavado de las aves y las vísceras y en los desagües de equipos como la escaldadora, el prechiller y los chillers; para mantener la limpieza y garantizar la calidad del producto, generándose aguas residuales que contienen altos niveles de materia orgánica medida como demanda química de oxígeno (DQO), sólidos suspendidos (SS), fósforo, nitrógeno, aceites y grasas ( $A$ y $G$ ), además del característico color rojizo por la presencia de sangre (Caldera y col., 2014; Rahman y col., 2014; Fernández y Betancourt, 2018; Nardi y col., 2011). Estas aguas son altamente contaminantes, cuando se disponen sin tratamiento, pueden agotar el oxígeno disuelto de un cuerpo de agua receptor, causar destrucción de la fauna, producir olores desagradables y facilitar el desarrollo y la propagación de enfermedades (Yaakob y col., 2018; Caldera y col., 2010).

Para mejorar la calidad de las aguas residuales de industrias avícolas (ARIA) se han propuesto varias operaciones y procesos unitarios. La selección de un tratamiento específico depende de las características de las aguas residuales, el costo del tratamiento existente y el cumplimiento de las regulaciones ambientales. Entre las tecnologías que pueden producir efluentes de alta calidad en las ARIA se encuentran: ósmosis inversa, flotación por aire disuelto (DAF) y lodos activados de película integrada fija (Baker y col., 2020). En este sentido, los sistemas híbridos que consisten en la combinación de dos (2) o más procesos unitarios biológicos y químicos y operaciones físicas de tratamiento para la eliminación más eficaz de los contaminantes de las aguas residuales, han ganado mucha atención en los últimos años, encontrándose disponibles los tratamientos fisicoquímicos (Tee y col., 2016; Vicuña y col., 2009). Un sistema híbrido lo representa la combinación 
de los métodos de flotación y coagulación, presentándose como alternativa para mejorar la calidad del agua en las etapas primarias del tratamiento.

En cuanto a la flotación, es uno de los sistemas de separación de fases más eficiente en el tratamiento de las aguas residuales provenientes de mataderos y consiste en separar sólidos de baja densidad - partículas líquidas de una fase líquida. Basado en los métodos de generación de las burbujas y los fundamentos del proceso, se han desarrollado diferentes unidades para inducir la flotación, entre las cuales se destacan: flotación por aire disuelto (DAF, por sus siglas en inglés), flotación por aire disperso, electroflotación, flotación por chorro o tecnología de celdas Jameson y flotación por ozono disperso (Ndikubwimana y col., 2016).

Entre los tipos de flotación más eficaces se encuentra la DAF cuya separación de los contaminantes se lleva a cabo introduciendo un gas (normalmente aire) en la fase líquida, en forma de burbujas. La fase líquida se somete a un proceso de presurización para alcanzar una presión de funcionamiento que oscila entre dos (2) a seis (6) atm, en presencia de suficiente aire para conseguir la saturación en aire del agua. Luego este líquido saturado de aire se somete a un proceso de despresurización llevándolo hasta la presión atmosférica por paso a través de una válvula reductora de presión (Salas y Condorhuamán, 2008).

Seguidamente, la despresurización permite que se formen pequeñas burbujas de aire que se desprenden de la solución. Los sólidos en suspensión o las partículas líquidas flotan, debido a que estas pequeñas burbujas se adhieren a los mismos y los obligan a elevarse a la superficie. Los sólidos en suspensión concentrados pueden separarse de la superficie por sistemas mecánicos. El líquido clarificado es separado $y$, parte del mismo puede reciclarse, para lograr mayores eficiencias de separación de contaminantes (Salas y Condorhuamán, 2008).

La eficiencia de los sistemas de DAF se ha demostrado durante el tratamiento de aguas residuales complejas, mostrado su eficiencia en la remoción de $A$ y $G$, hidrocarburos, SS, DQO, turbidez y color (Sena y col., 2008; Fernández y col., 2015; Miranda y col., 2013). Adicionalmente, la efectividad de un sistema de DAF podría incrementarse agregando coagulantes $y$ mejorando las condiciones de presurización y recirculación (Dassey y Theegala, 2012; Nardi y col., 2011 ; Younker y Walsh, 2014; Caldera y col., 2014; Del Nery y col., 2016; Herrera y col., 2014). Entre los coagulantes utilizados para remover contaminantes de las aguas residuales de mataderos se encuentran: sulfato de aluminio, cloruro férrico, sulfato férrico, policloruro de aluminio, quitosano, poliacrilamida, polímeros aniónicos, biopolímeros, biofloculantes, combinaciones de coagulante y floculantes, entre otros (Bustillos y Mehvar, 2015; López y col., 2008; Caldera y col., 2014; Dlangamandla y col., 2018).

El sistema de DAF permite remover en menor tiempo las partículas pequeñas $y$ ligeras como los A y G (Edzwald, 2010; Shammas y Bennett, 2010; Dassey y Theegala, 2012; Miranda y col., 2013). Los A y $G$ deben ser eliminados eficientemente durante el tratamiento primario puesto que su presencia en altas cantidades en las aguas residuales provocan averías en las bombas, obstrucciones y la formación de capas flotantes, colaboran con el desarrollo 
de organismos filamentoso y dificultan el tratamiento (Yoo y Hsieh, 2010; Weihua y Mehrab, 2011 ; Del Nery y col., 2007).

Por lo anteriormente expuesto, esta investigación tiene como objetivo evaluar la eficiencia de la aplicación de policloruro de aluminio (PAC) en un sistema de flotación por aire disuelto (DAF) a escala piloto, para el tratamiento de aguas residuales de una industria avícola.

\section{MÉTODO}

\section{Toma de muestras}

Las muestras de agua residual se recolectaron manualmente, durante seis meses de evaluación, en la entrada de una unidad de flotación por aire disperso, que integra el sistema de tratamiento de una industria avícola ubicada en el estado Zulia, Venezuela. Esta unidad de tratamiento ha presentado problemas de funcionamiento, removiendo bajas porcentajes de A y G. Las características de las ARIA se presentan en la Tabla 1. Por esta razón, se planteó la sustitución o acondicionamiento de esta unidad de flotación (flotación por aire disperso) por un sistema de DAF, como alternativa para remover $A$ y $G$, evitar problemas en las unidades posteriores y mejorar la eficiencia del sistema de tratamiento de las ARIA.

El sistema de tratamiento de la industria avícola está conformado por las siguientes unidades: tamiz rotatorio, unidad de flotación de $A$ y $G$, lodos activados (reactor biológico y sedimentador secundario) y cámara de cloración. En cuando a las unidades para el manejo de lodo, consta de un digestor aerobio, espesador y lechos de secado. A este sistema de tratamiento entran las aguas residuales provenientes de las diferentes áreas de la matanza de aves y el proceso productivo en general, después de separar o recuperar las plumas, sangre y vísceras (Caldera y col., 2010).

Tabla 1. Características de las aguas residuales de la industria avícola

\begin{tabular}{llll}
\hline Parámetro & Máximo & Mínimo & Promedio \pm Desviación \\
\hline DQO $(\mathrm{mg} / \mathrm{l})$ & $4.740,0$ & $1.135,0$ & $2.446,6 \pm 624,6$ \\
$\mathrm{~A} \mathrm{y} \mathrm{G}(\mathrm{mg} / \mathrm{l})$ & 570,9 & 340,9 & $485,1 \pm 91,2$ \\
$\mathrm{SST}(\mathrm{mg} / \mathrm{l})$ & 718,0 & 330,0 & $542,6 \pm 115,3$ \\
$\mathrm{pH}$ & 7,0 & 6,6 & $6,8 \pm 0,2$ \\
\hline
\end{tabular}

\section{Equipo experimental}

A continuación se describe el equipo experimental de flotación por aire disuelto a escala de laboratorio utilizado en esta investigación (Figura 1). El equipo de DAF tiene una cámara de flotación (1) con capacidad para $4 \mathrm{l}$, de fondo perforado para distribuir uniformemente el reciclo y aumentar el contacto gas-líquido, una válvula que regula la entrada del agua presurizada (2), y una válvula de salida (3) para la muestra tratada. También tiene un tanque de presurización de acero inoxidable con capacidad de 2 I (4), con un manómetro en la parte superior (5) para verificar la presión interna, un recipiente de llenado para introducir el reciclo (6) y una válvula (7) que permite la entrada del 
reciclo. En la parte inferior posee dos válvulas, la primera (8) permite el paso del aire proveniente del compresor de aire (9), y la segunda (10) libera el líquido presurizado; esta misma válvula es usada para la succión del aire del tanque a través de una bomba de succión (11), tiene además conexiones flexibles con acoples de rápida instalación y desinstalación.

\section{Procedimiento experimental}

Para realizar los ensayos en el sistema de $D A F$, se seleccionaron diferentes condiciones de operación, en cuanto a presión, recirculación y adición de coagulante. Las presiones evaluadas fueron $207 \mathrm{kPa}$ (30 psi), $276 \mathrm{kPa}(40$ psi) y $345 \mathrm{kPa}(50$ psi), variando el porcentaje de recirculación del efluente en $20 \%$, $30 \%$ y $40 \%$, trabajando $\sin y$ con la adición del coagulante policloruro de aluminio (PAC). En el caso de las pruebas con PAC se trabajó con una solución al $25 \%$ p/v, preparando una solución patrón de $10000 \mathrm{mg} / \mathrm{l}$, probando las concentraciones de 220, 240, 260, 280 y $300 \mathrm{mg} / \mathrm{l}$ en pruebas de coagulación y seleccionando la concentración óptima de $240 \mathrm{mg} / \mathrm{l}$.

Se iniciaron las pruebas en el sistema de DAF haciendo succión en el tanque hasta obtener el vacío, luego se agregó el agua residual y se sometió a la presión deseada. Seguidamente, se conectó la cámara y se descargó la muestra ya presurizada. Se dejó en reposo por 10 minutos, para llevar a cabo el proceso de flotación. Finalmente, se tomaron las muestras requeridas para determinar los parámetros fisicoquímicos. El procedimiento fue similar cuando se agregó el coagulante, se trabajó con una mezcla rápida por 1 min, luego una mezcla lenta por 10 minutos, con la concentración óptima del coagulante PAC, repitiendo por triplicado cada ensayo.

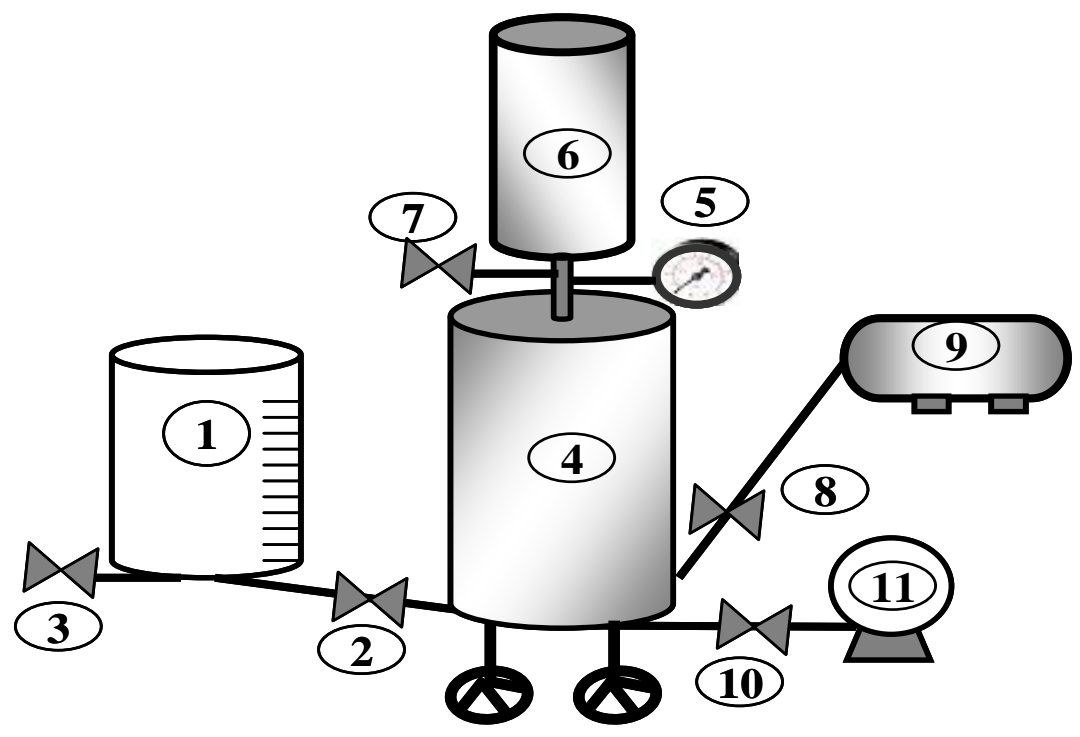

Figura 1. Equipo de flotación por aire disuelto (DAF) utilizado en esta investigación. 
La eficiencia del sistema de DAF se evaluó determinando las concentraciones de $A$ y $G$ antes y después del tratamiento. Adicionalmente se midieron los parámetros DQO y sólidos suspendidos totales (SST), según métodos estándar APHA y col. (1998). Las concentraciones de $A$ y $G$ se determinaron por duplicados, mientras que el resto de los parámetros se realizaron por triplicados.

\section{RESULTADOS}

En la Figura 2, se presentan los porcentajes de remoción de $A$ y $G$, durante las pruebas de flotación en el sistema de DAF, a las presiones de 30, 40 y 50 psi, con recirculaciones de 20, 30 y $40 \%$, sin la adición del coagulante PAC. Se observa que para la condición $20 \%$ de reciclo y 40 psi se obtiene la mayor remoción de A y G (97\%), mientras que la menor remoción se obtuvo para la condición $40 \%$ de reciclo y presión de 50 psi (87\%). También se visualiza que la eficiencia del sistema fue mayor a la presión de 40 psi, disminuyendo con el aumento de la recirculación del efluente. El incremento del reciclo también disminuyó la eficiencia a la presión de 50 psi.

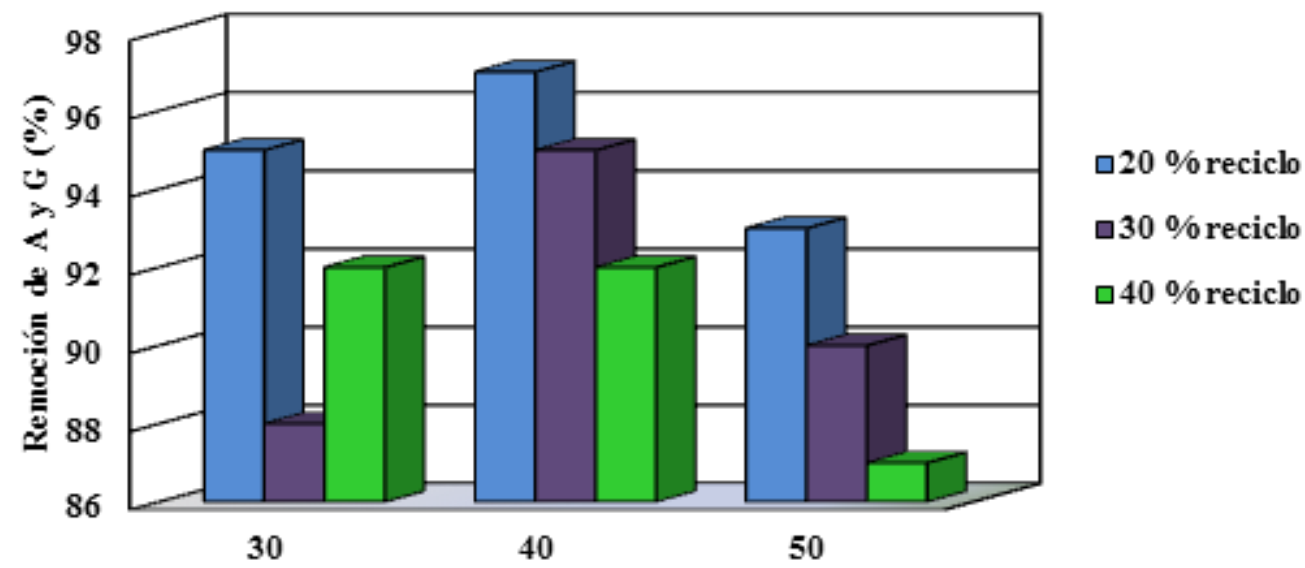

Presión (psi)

Figura 2. Comportamiento de la remoción de $A$ y $G$ en el sistema DAF, sin adición de coagulantes.

Para las diferentes presiones evaluadas, el sistema de DAF logra remover más del $92 \%$ de los A y $G$ presentes en las ARIA cuando se recirculó el $20 \%$ del efluente (Figura 2). AlMutairi y col. (2008), obtuvieron remociones menores de $A$ y $G(84 \%)$ en un sistema DAF, trabajando a mayor presión (150 psi) y menor recirculación (15\%), durante el tratamiento de aguas residuales de matadero.

En cuanto a la remoción de DQO, a las diferentes condiciones de operación del DAF, sin la adición de PAC; se obtuvo una remoción máxima de 51 \% (Tabla 2), correspondiente a la condición de $20 \%$ de reciclo y 50 psi. Para la presión de 40 psi y los diferentes reciclos, la remoción de la DQO fue superior al $35 \%$, considerándose apropiada para unidades de tratamiento primario, puesto que se mejora la eficiencia de las unidades posteriores (Mittal, 2006; Sena y col., 2008). El incremento del reciclo 
causó la disminución de la eficiencia del sistema de DAF a altas presiones (50 psi).

Asimismo, en la Tabla 2 se observan remociones de SST inferiores al $50 \%$ para las diferentes condiciones de presión y recirculación evaluadas. La mayor remoción
(47\%) se obtuvo con las condiciones $20 \%$ de reciclo y de 50 psi. El aumento de la presión favoreció la eficiencia del sistema de DAF para remover SST, al $30 \%$ de recirculación.

Tabla 2. Remoción A y G, DQO y SST en el sistema de DAF, sin adición de coagulantes.

\begin{tabular}{ccccc}
\hline $\begin{array}{c}\text { Reciclo } \\
\text { (\%) }\end{array}$ & $\begin{array}{c}\text { Presión } \\
\text { (psi) }\end{array}$ & $\begin{array}{c}\text { Remoción de A y G } \\
\text { (\%) }\end{array}$ & $\begin{array}{c}\text { Remoción de DQO } \\
\text { (\%) }\end{array}$ & $\begin{array}{c}\text { Remoción de SST } \\
\text { (\%) }\end{array}$ \\
\hline & 30 & 95 & 24 & 33 \\
20 & 40 & 97 & 40 & 25 \\
& 50 & 93 & 51 & 47 \\
& 30 & 88 & 44 & 41 \\
30 & 40 & 95 & 36 & 42 \\
& 50 & 90 & 35 & 44 \\
& 30 & 92 & 40 & 18 \\
& 40 & 92 & 38 & 32 \\
\hline
\end{tabular}

Según estos resultados, seleccionando las condiciones de presión y reciclo $(20 \%$ de reciclo y 40 psi) que favorecieron la eficiencia del sistema de DAF para remover el $97 \%$ de los $A$ y $G$ presentes en las ARIA, las remociones de DQO y SST alcanzaron $40 \%$ y $25 \%$, respectivamente. El PAC como coagulante ha mostrado eficiencia en la remoción de elevadas concentraciones de $A$ y $G$ presentes en aguas residuales. Ahmad y col. (2006) reportan remociones superiores a $80 \%$ de aceites cuando compararon su efectividad con la del sulfato de aluminio. Concluyeron que el PAC requirió menores dosis para remover SS y aceites que el sulfato de aluminio, debido a las propiedades del
PAC como alta densidad de carga, por lo que su uso se ha vuelto más común.

Se puede inferir que la adición del coagulante favorecería solo la remoción de estos dos parámetros, puesto que su eficiencia quedó demostrada para remover A y $G$ sin la ayuda de coagulantes. Por otra parte, si se seleccionan las condiciones 20 $\%$ de reciclo y $50 \mathrm{psi}$, donde se obtuvo la mayor remoción de los otros parámetro evaluados en esta investigación, se obtiene $93 \%, 51 \%$ y $47 \%$ de A y G, DQO y SST, respectivamente. En los dos casos, la adición del coagulante disminuiría la carga contaminante de DQO y SST presentes en las ARIA. 
Otros autores han reportado la eficiencia de sistemas de DAF a diferentes condiciones de presión y recirculación. Del Nery y col. (2007), obtuvieron una remoción A y $G$ del $57 \%$, menor a la encontrada en esta investigación, en un sistema de DAF, sin adición de coagulantes y con presurización completa del efluente cárnico. Mientras que, Salas y Condorhuamán (2008) probaron varios porcentajes de recirculación y encontraron la máxima eficiencia con recirculación del $100 \%$. Concluyeron que la flotación con aire disuelto permite reducir la carga contaminante contenida en los efluentes generados en un matadero, removiendo la DQO en $75 \%$ y los $A$ y $G$ en $95 \%$.

Después de los resultados anteriores se continuó con la adición del coagulante
PAC, a la concentración de $240 \mathrm{mg} / \mathrm{L}$ y las condiciones de operación propuestas, presiones de 30,40 y 50 psi y los reciclo de $20 \%$, $30 \%$ y $40 \%$. En la Figura 3 se muestra el comportamiento de la remoción de $A$ y $G$ en el sistema de DAF con PAC, a las diferentes condiciones, donde se puede apreciar que las remociones de $A$ y $G$ variaron en un rango de $91 \%$ a $97 \%$, después del tratamiento. La mayor remoción de $A$ y $G(97 \%)$ se obtuvo con la condición de operación de 50 psi y $40 \%$. Adicionalmente, se observa en Figura 3 que el aumento del porcentaje de recirculación mejora la eficiencia del sistema de DAF a presiones más elevadas (50 psi).

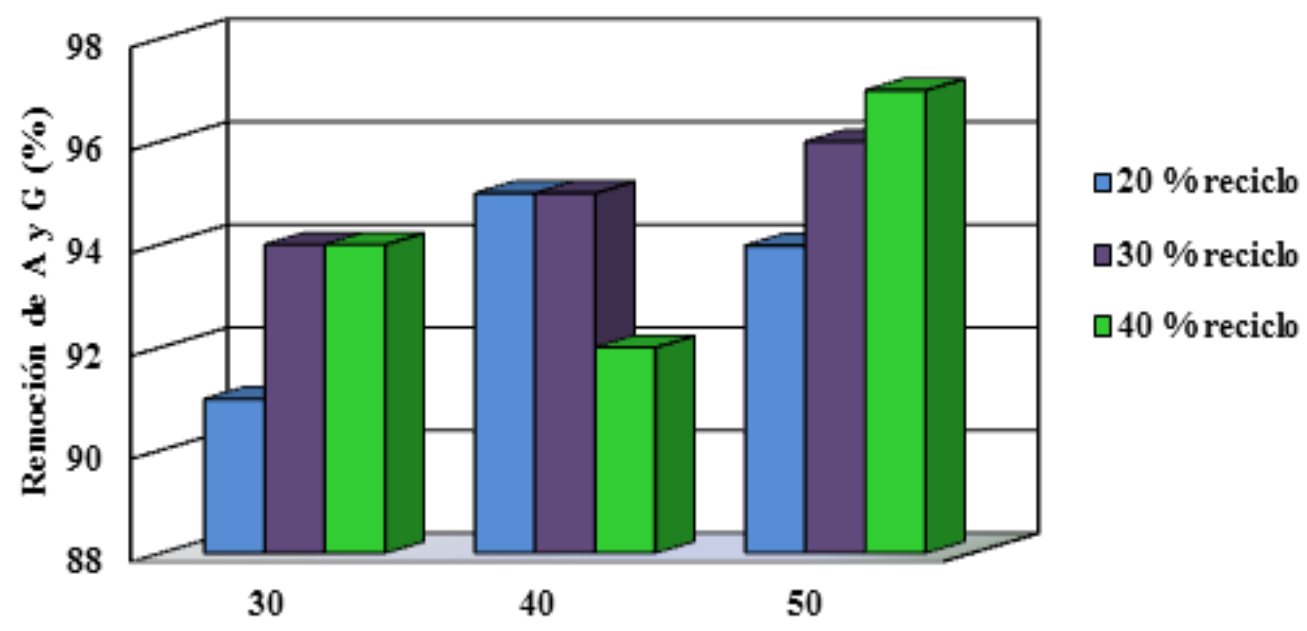

Presión (psi)

Figura 3. Comportamiento de la remoción de $A$ y $G$ en el sistema DAF, con adición de PAC como coagulante.

Con respecto a la remoción de DQO se observa en la Figura 4, que la adición de PAC incrementó la eficiencia del sistema de DAF para remover este parámetro de las ARIA. La máxima remoción de DQO (90\%) se obtuvo para la condición de 50 psi y $40 \%$ de recirculación, mientras que la condición menos favorable fue 50 psi y 
$20 \%$ de reciclo (79 \%). No se observó tendencia regular entre las diferentes condiciones de presión, sin embargo, al aumentar el porcentaje de recirculación para la presión de 50 psi mejoró la eficiencia de remoción de DQO. Si se comparan los resultados del sistema de DAF con y sin PAC, se obtiene un incremento en la remoción de DQO de aproximadamente $40 \%$. Resultados similares reportaron Poh y col. (2014), quienes trabajaron por flotación con microburbujas durante el tratamiento de efluentes de plantas de aceite de palma, logrando eficiencia de DQO de 53,7\%, mientras que, al agregar $2 \mathrm{~g} / \mathrm{L}$ de PAC, la eliminación de DQO alcanzó el $93 \%$.

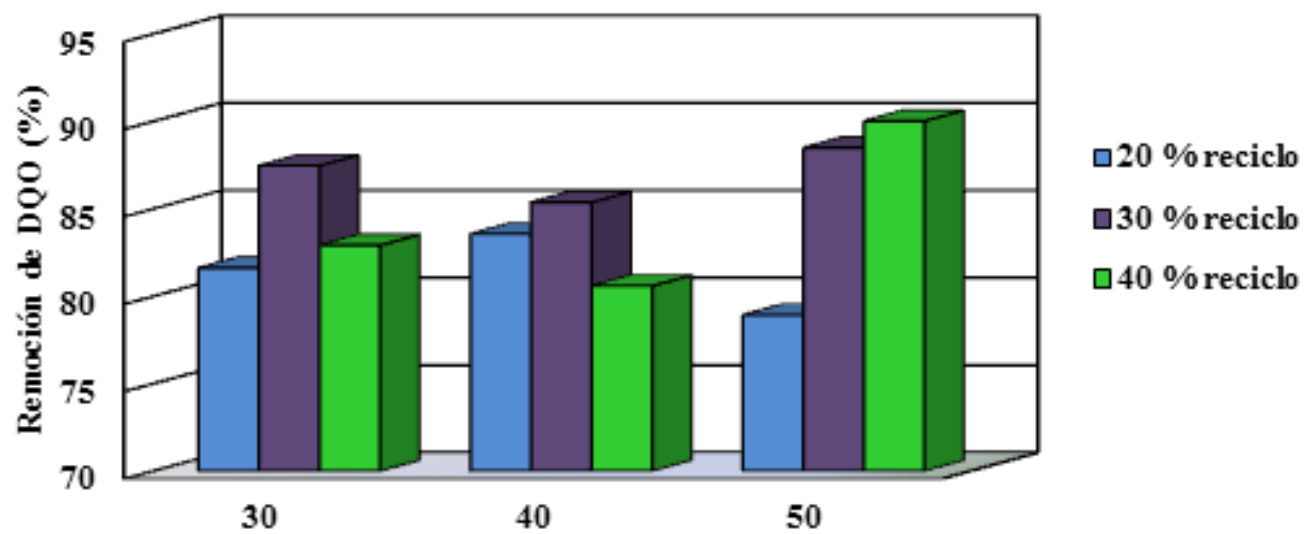

Presión (psi)

Figura 4. Comportamiento de la remoción de DQO en el sistema DAF, con adición de PAC como coagulante.

En cuanto al análisis de los SST, se aprecia en la Figura 5 que a las diversas condiciones de operación, el sistema de DAF con PAC removió entre 51 y $79 \%$ de SST, lo que representa un aumento de la remoción de SST con respecto al sistema sin coagulante. Se observó una tendencia al aumento de la remoción de SST al incrementarse la presión para el porcentaje de reciclo de $30 \%$, mientras que para el reciclo de $20 \%$ la tendencia es a disminuir. A las condiciones de 50 psi y $40 \%$ de reciclo, donde se obtuvo la mayor remoción del parámetro de interés $(A$ y $G$ ) con PAC, se observa una remoción, $60 \%$ de SST.

La eficiencia de remoción de A y G, SS y DQO ha sido evaluada por otros autores, quienes han presentado resultados de investigaciones realizadas en sistemas de
DAF con diversos coagulantes y aguas residuales industriales, variando las condiciones de operación de presión y recirculación. Dassey y Theegala (2010), trabajaron en un sistema de DAF recirculando el $40 \%$ de ARIA con una presión mayor a la evaluada en esta investigación (90 psi), consiguieron remociones altamente eficientes de $\mathrm{A}$ y $\mathrm{G}$ y DQO de $99 \%$ y del $96 \%$ para los SST, emplearon una combinación de $800 \mathrm{mg} / \mathrm{L}$ de cloruro férrico y $900 \mathrm{mg} / \mathrm{L}$ de un polímero. Mientras que Al-Shamrani y col. (2002), evaluaron la eficiencia de un DAF con recirculaciones del $10 \%$ y operado a 80 psi, adicionaron $100 \mathrm{mg} / \mathrm{L}$ del coagulante sulfato de aluminio a aguas aceitosas, reportaron $99,3 \%$ de remoción de $A$ y $G$. 


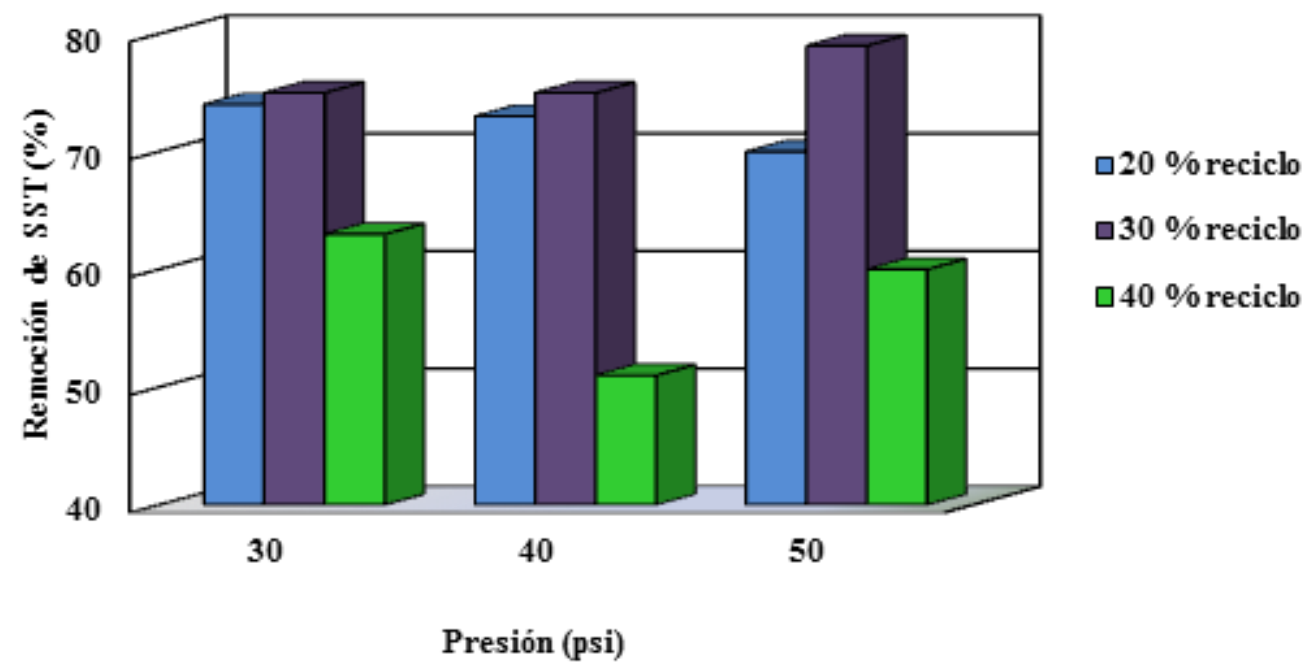

Figura 5. Comportamiento de la remoción de SST en el sistema DAF, con adición de PAC como coagulante.

De esta manera el PAC logró aumentar la remoción de DQO y SST presentes en las AIRA, para las distintas condiciones de operación, obteniendo valores máximos de $90 \%$ y $79 \%$, respectivamente, sin causar cambios importantes en la alta remoción de A y G (97\%) lograda sin la adición de coagulante. En la Figura 6 se muestra el comportamiento para los tres parámetros evaluados ( $A$ y $G, D Q O$ y SST) después del tratamiento con PAC cuando la recirculación del efluente se mantuvo en $30 \%$ y se variaron las presiones, observando poca variación en las remociones obtenidas a las diferentes presiones. A esta condición de recirculación se obtuvieron remociones superiores al $75 \%$, considerando la presión de 50 psi como la más favorable con remociones de 96, 89 y $79 \%$ para Ay G, DQO y SST, respetivamente. Por su parte, Caldera y col. (2014) reportaron como condición de operación óptima 40 psi y 40 $\%$ de recirculación, durante el tratamiento de ARIA con altas concentraciones de $A$ y $G$ (1905 mg/L) en el mismo equipo de DAF evaluado en esta investigación.

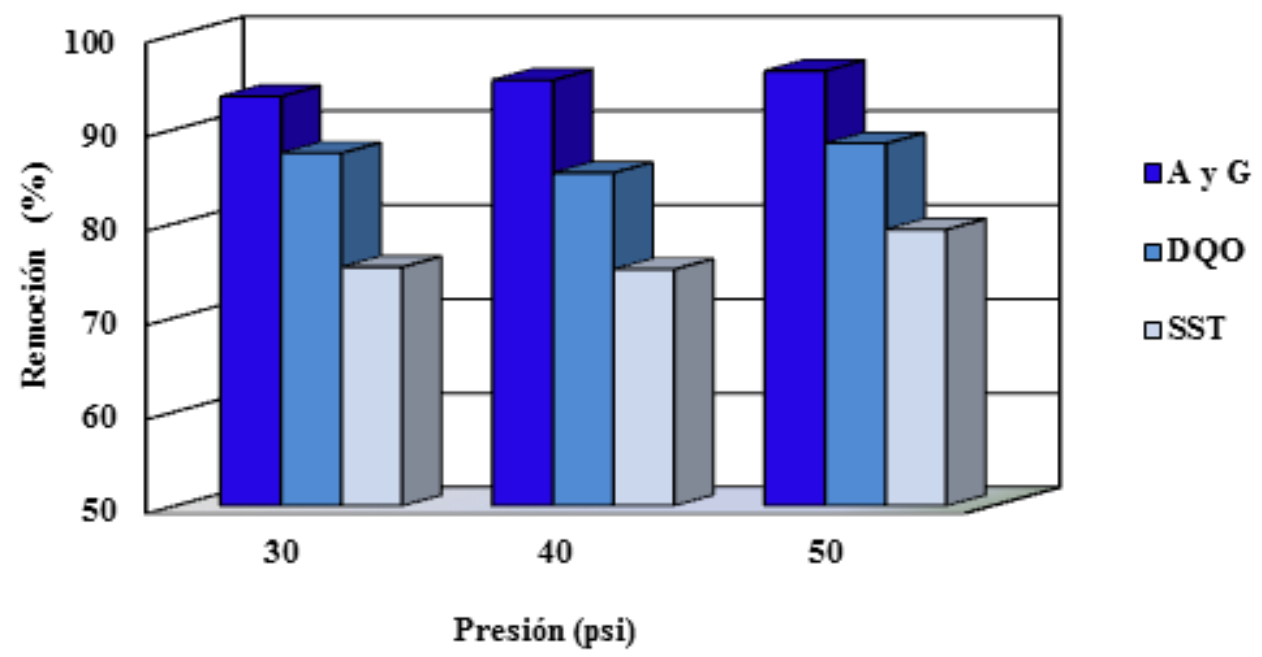

Figura 6. Comportamiento de los parámetros Ay G, DQO y SST a diferentes presiones y recirculación de $30 \%$, en el sistema DAF, con adición de PAC como coagulante. 
Díaz y col. (2018) lograron disminuir la carga contaminante de las aguas residuales de una refinería utilizando un DAF, con la adición de PAC como coagulante $(200 \mathrm{mg} / \mathrm{l})$ más un floculante (3 $\mathrm{mg} / \mathrm{L})$ y variaciones de las condiciones de operación de presión (58 a 72,5 psi) y recirculación (10 a 50 \%). Reportan resultados similares en la selección de las condiciones óptimas de operación, recirculación del 30 \% y la mayor presión evaluada (72,5 psi), con estas condiciones lograron las mayores remociones, 93,88\% de SS y $95,35 \%$ para hidrocarburos. De la misma manera, Nardi y col. (2008), presentaron resultados bastante similares cuando evaluaron la eficiencia de un DAF a escala de laboratorio durante el tratamiento de ARIA, trabajaron con $40 \%$ de recirculación y 65,3 psi (450 kpa), agregando PAC como coagulante combinado con un polímero aniónico como floculante, encontraron eficiencias de remoción de SS y A y G de 74 \% y 99 \%, respectivamente.

\section{CONCLUSIONES}

Los resultados obtenidos evidencian la eficiencia del sistema de DAF, sin y con la adición del coagulante PAC a las ARIA, alcanzando remociones de $A$ y $G$ máximas del $97 \%$. Sin embargo, la respuesta fue diferente para el resto de los parámetros evaluados, donde la adición del coagulante permite un aumento en la remoción de los parámetros DQO (90 \%) y SST (79\%).

A las condiciones de operación óptimas, recirculación de $40 \%$ y presión 50 psi, con la adición del coagulante PAC a las ARIA, se obtuvieron las mayores remociones de $A$ y $G$ y DQO, 97 y $90 \%$ y mientras que los SST alcanzaron $60 \%$.

El sistema de flotación con aire disuelto (DAF) con la adición de policloruro de aluminio (PAC) representa una alternativa para mejorar la calidad de las aguas residuales provenientes de la industria avícola (ARIA), lo que podría facilitar el tratamiento y acortar los tiempos de retención en las unidades posteriores del sistema de tratamiento.

\section{Agradecimiento}

Al Consejo de Desarrollo Científico y Humanístico de la Universidad del Zulia (CONDES LUZ) por el financiamiento a este proyecto.

\section{REFERENCIAS}

Ahmad, A., Sumathi, S., y Hameed, B. (2006). Coagulation of residue oil and suspended solid in palm oil milk effluent by chitosan, alum and PAC. Chemical Engineering Journal 118(1-2), 99-105

Al-Mutairi, N., Al-Sharifi, F., y AlShammari, S. (2008). Evaluation study of a slaughterhouse wastewater treatment plant including contactassisted activated sludge and DAF. Desalination 225, 167-175

Al-Shamrani, A., Jamesa, A., y Xiao, H. (2002). Destabilisation of oil-water emulsions and separation by dissolved air flotation. Water Research 36, 15031512

APHA, AWWA, WCF (1998). En: Standard methods for examination of water and wastewater. 18th Ed. Washington DC, USA

Baker, B., Mohamed, R., Al-Gheethi, A., y Aziz, H. (2020). Advanced technologies for poultry slaughterhouse wastewater treatment: A systematic review. Journal of Dispersion Science and Technology. 
doi.org/10.1080/01932691.2020.172 1007

Bustillos, C., y Mehvar, M. (2015). Slaughterhouse characteristics, treatment, and management in the processing industry: A review on trends and advances. Journal of Environmental Management 161, 287-302

Caldera, Y., Gutiérrez, E., Albarrán, H., Navarro, P., y Sánchez, M. (2014). Quitosano como coagulante en un sistema de flotación durante el tratamiento de aguas residuales de un matadero de aves. Impacto Científico 9 (2), 215-227

Caldera, Y., Gutiérrez, E., Luengo, M., Chávez, J., y Ruesga, L. (2010). Evaluación del sistema de tratamiento de aguas residuales de industria avícola. Revista Científica FCV-LUZ, XX(4), (2010), 409-416

Dassey, A., y Theegala, C. (2012). Evaluating coagulation pretreatment on poultry processing wastewater for dissolved air flotation. Journal of Environmental Science and Health 47(13), 2069-2076

Del Nery, V., Damianovic, M., Moura, R., Pozzi, E., Pires, E., y Foresti, E. (2016). Poultry slaughterhouse wastewater treatment plant for high quality effluent. Water Science \& Technology 73(2), 309-316

Del Nery, V., Nardi, I., Damianovic, M., Pozzi, E., Amorim, A., y Zaiat, M. (2007). Long-term operating performance of a poultry slaughterhouse wastewater treatment plant. Resources Conservation \& Recycling 50 (1): 102-114

Díaz, M., Rivas, L., Fernández, D., Salazar, D., y Miller, S. (2018). Tratamiento de aguas residuales oleosas mediante flotación por aire disuelto. Tecnología Química 8(2), 256-270

Dlangamandla, C., Ntwampe, S., y Basitere, M. (2018). A bioflocculant- supported dissolved air floatation system for the removal of suspended solids, lipids and protein matter from poultry slaughterhouse wastewater. Water Sci. \& Technol. 78(2), 452-458

Edzwald, J. (2010). Dissolved air flotation and meat. Water Research 44, 2077 . 2106

Fernández, A., y Betancourt, A. (2018). Destino sostenible de los residuos generados en las plantas de beneficio avícola. Aibi Revista de Investigación, Administración e Ingeniería 6(2), 11-22

Fernández, D., Súarez, A. Díaz, M., Rivas, L., Cañete, C., Romero, R., Teuteló, R., Miller, S., y La Maza, N. (2015). Obtención de parámetros óptimos de operación de flotación por aire disuelto. Revista CENIC Ciencias Químicas 46, 33-44

Herrera, L., Flores, P., Mejías, P., Vargas, L., Cárdenas, C., Araujo, I., Del Villar, N., y Delgado, J. (2014). Tratamiento de aguas residuales domésticas para su potencial reutilización industrial. Boletín del Centro de Investigaciones Biológicas 48(2), 101-114

López, A., De La Barrera, J., Vallejo, R., y Barahona, C. (2008). Estudio comparativo entre un proceso fisicoquímico y uno biológico para tratar agua residual de rastro. Interciencia 33(7), 490-495

Meleán, R., Bonomie, M., y Rodríguez, G. (2008). Procesos productivos de la industria avícola zuliana: Fases alimento, engorde y beneficio. Rev. Fac. Agron. (LUZ). 25, 160-184

Miranda, R., Nicu, R., Latour, I., Lupei, M., Bobu, E., y Blanco, A. (2013). Efficiency of chitosans for the treatment of papermaking process water by dissolved air flotation. Chemical Engineering Journal 231, 304-313

Mittal, G. (2006). Treatment of wastewater from abattoirs before land application-a review. Bioresource Technology 97, 1119-1135 
Nardi, I., Del Nery, V., Amorim, A., Dos Santos, N., y Chimenes, F. (2011). Performances of SBR, chemical-DAF and UV disinfection for poultry slaughterhouse wastewater reclamation. Desalination 269(1-3), 184-189

Nardi, I., Fuzi, T., y Del Nery, V. (2008). Performance evaluation and operating strategies of dissolved-air flotation system treating poultry slaughterhouse wastewater. Resources, Conservation and Recycling 52(3), 533-544

Ndikubwimana, T., Chang, J., Xiao, Z., Shao, W., Zeng, X., Ng, I., y Lu, Y. (2016). Review. Flotation: A promising microalgae harvesting and dewatering technology for biofuels production. Biotechnol. J. 11, 315-326

Poh, P., Ong, W., Lau, E., y Chong, M. (2014). Investigation on micro-bubble flotation and coagulation for the treatment of anaerobically treated palm oil mill effluent (POME). J. Environ. Chem. Eng. 2(2), 1174-1181

Rahman, U., Sahar, A., y Khan, M. (2014). Recovery and utilization of effluents from meat processing industries. Food Research International 65(C), 322-328

Salas, G., y Condorhuamán, C. (2008). Tratamiento de las aguas residuales de un centro de beneficio o matadero de ganado. Rev. Per. Quím. Ing. Quím. $11(1), 29-35$

Sena, R., Moreira, R., y José, H. (2008). Comparison of coagulants and coagulation aids for treatment of meat processing wastewater by column flotation. Bioresource Technology 99(17), 8221-8225
Shammas, N., y Bennett, G. (2010) Principles of air flotation technology. Handbook of Environmental Engineering. Flotation Technology 12, $1-47$

Tee, P., Abdullah, M., Tan, I., Rashid, N., Amin, M., Nolasco-Hipolito, C., y Bujang, K. (2016). Review on hybrid energy systems for wastewater treatment and bio-energy production. Renewable and Sustainable Energy Reviews 54(2), 235-246

Vicuña, E., Silva, L., Rojas, A., y Loayza, J. (2009). Sistemas híbridos de tratamiento de aguas residuales. Rev. Per. Quim. Ing. Quim. 12(1), 10-17

Weihua, C., y Mehrab, M. (2011). Slaughterhouse wastewater treatment by combined anaerobic baffled reactor and $\mathrm{UV} / \mathrm{H}_{2} \mathrm{O}_{2}$ processes. Chemical Engineering Research and Design 89(7), 1136-1143

Yaakob, M., Mohamed, R., Al-Gheethi A., y Kassim, A. (2018). Characteristics of chicken slaughterhouse wastewater. Chemical Engineering Transactions 63, 637-642. doi:10.3303/CET1863107

Yoo, S., y Hsieh, J. (2010) Advanced water recycling through electrochemical treatment of effluent from dissolved air flotation unit of food processing industry. Water Science \& Technology 61(1), 181-190

Younker, J., y Walsh, M. (2014) Benchscale investigation of an integrated adsorption-coagulation-dissolved air flotation process for produced water treatment. Journal of Environmental Chemical Engineering 2, 692-697 